\title{
Comparison of Glycemic Status and Insulin Resistance before and after Sleeve Gastrectomy in Morbid Obese Patients with Type 2 Diabetes Mellitus
}

\author{
Mukesh K Meena ${ }^{1}$, Charu Yadav², Meena Varma ${ }^{3}$, Anju Jain ${ }^{4}$
}

\begin{abstract}
Background: Obesity has emerged as one of the most serious public health concerns in the twenty-first century. Currently, bariatric surgeries are considered to be the only successful, long-term therapy available for weight loss in morbid obese subjects. Recent research has focused on sleeve gastrectomy (SG) as a "cure" for comorbid conditions like diabetes mellitus.

Materials and methods: This hospital-based prospective study was conducted in 50 morbid obese subjects with type 2 diabetes mellitus (T2DM) aged 20-40 years to evaluate glycemic indices and insulin resistance before and after SG at 9 and 12 weeks. Fasting plasma glucose was estimated using the glucose oxidase peroxidase method and serum insulin by the electrochemiluminescence method in automated analyzers. Insulin resistance was calculated using the homeostatic model assessment of insulin resistance (HOMA-IR) calculator. Data analysis was done using SPSS ver. 18 employing the ANOVA and Tukey's post hoc test.

Results: A total of 50 cases were included in the study (19 women, 31 men; aged $20-40$ years; duration of diabetes $<10$ years). Fasting plasma glucose and HOMA-IR decreased significantly $(p<0.05)$ at 9 and 12 weeks follow-up after SG.

Conclusion: The present study conducted in morbid obese patients with T2DM highlights the fact that SG procedure showed early beneficial effects on the glycemic status as well as an improvement in the insulin sensitivity rather than an increased insulin secretion.

Clinical significance: The improved glycemic status in this study along with an understanding of the underlying mechanisms could not only provide an insight into the pathophysiology of "diabesity" but also assist in the development of a better therapeutic approach for long-term remission of diabetes in obese patients.
\end{abstract}

Keywords: Bariatric surgery, Insulin resistance, Obesity, Prospective study, Sleeve gastrectomy, Type 2 diabetes.

Indian Journal of Medical Biochemistry (2019): 10.5005/jp-journals-10054-0126

\section{INTRODUCTION}

The rapidly increasing prevalence of obesity, with morbid obesity affecting about $5 \%$ of India's population, has become a major public health concern. ${ }^{1}$ Studies have indicated that obesity is responsible for more than 2.8 million deaths worldwide per year owing to an increased prevalence of its comorbidities like type 2 diabetes mellitus (T2DM), hyperlipidemia, hypertension, etc. ${ }^{2,3}$

Literature data have shown that weight loss after pharmacotherapy or diet restrictions are not very effective in the long run. ${ }^{4}$ In such a scenario, bariatric surgeries intended for weight loss seem to fill the gap. In addition to weight loss, studies have shown remarkable diabetes remission in short- to medium-term follow-up, particularly after Roux-en-Y gastric bypass and SG. ${ }^{5-9}$ The benefits of SG, one of the restrictive bariatric methods, include not only food intake control as a consequence of reduced gastric volume but also influences gut hormones like insulin, glucagon-like peptide 1 (GLP-1), and ghrelin, which control energy metabolism..$^{10,11}$

Given the unrelenting diabetes crisis with more than $60 \%$ of diabetics being obese, ${ }^{12}$ new efficient therapies for management of this combination of diabetes and obesity, "diabesity," fetch the interest of researchers and clinicians alike. ${ }^{9}$ Keeping this in mind, the present study was taken up to estimate and compare the changes in the glycemic status and insulin resistance in morbid obesity T2DM patients undergoing SG.

\section{Materials and Methods}

A hospital-based prospective study was conducted in 50 morbid obese patients $\left(\mathrm{BMI}>35 \mathrm{~kg} / \mathrm{m}^{2}\right)^{2}$ of either sex aged $20-40$ years
1,2,4 Department of Biochemistry, Lady Hardinge Medical College and Smt Sucheta Kriplani Hospital, New Delhi, India

${ }^{3}$ Department of Biochemistry, Sri Aurobindo Institute of Medical Sciences and Medical College, Indore, Madhya Pradesh, India

Corresponding Author: Charu Yadav, Department of Biochemistry, Lady Hardinge Medical College and Smt Sucheta Kriplani Hospital, New Delhi, India, e-mail: drcharuy@gmail.com

How to cite this article: Meena MK, Yadav C, Varma M, et al. Comparison of Glycemic Status and Insulin Resistance before and after Sleeve Gastrectomy in Morbid Obese Patients with Type 2 Diabetes Mellitus. Indian J Med Biochem 2019;23(3):331-334.

Source of support: Nil

Conflict of interest: None

diagnosed with T2DM of less than 10 years duration who agreed to undergo bariatric surgery willingly. These patients were taken from the Metabolic and Bariatric Surgery Department of SAIMS Hospital during a period of 1 year. Detailed clinical assessment including medical history, clinical examination, and comorbidity evaluation was done by a multidisciplinary consulting team followed by estimation of fasting plasma glucose and insulin resistance at baseline (before surgery) and compared with the status after surgery, i.e., at 9 weeks and 12 weeks follow-up visits post SG. Patients with diabetes of more than 10 years duration, hypo- or hyperthyroidism, Cushing syndrome, history of convulsions, liver and kidney disease, ischemic heart disease, rheumatoid arthritis, chronic alcoholism, and pregnant women were excluded from 
this study along with those on hormonal contraceptives, diuretics and herbal treatment, and patients who had undergone bariatric surgery in the past. All procedures performed in this study were approved by the Institutional Ethics Committee and in accordance with the 1964 Helsinki Declaration and its later amendments. A written informed consent was obtained from all the subjects before enrolment into the study.

\section{Biochemical Analysis}

Serum from fasting venous blood samples was separated by centrifugation $(4,000 \mathrm{rpm}$ for 10 minutes) within 30 minutes of collection into vacutainers and stored at $-20^{\circ} \mathrm{C}$ till further analysis. Fasting plasma glucose were estimated on a daily basis by the glucose oxidase peroxidase method in a HITACHI-902 automated analyzer. Serum insulin estimation was done using electrochemiluminescence immunoassay in an ELECSYS-2010 analyzer. Insulin resistance was then computed using the homeostasis model assessment (HOMA) calculator.

\section{Statistical Analysis}

Statistical analysis was done using SPSS ver. 18 employing ANOVA for intergroup comparison of means with Tukey's as the post hoc test. $p<0.05$ was considered statistically significant.

\section{ResUlts}

Following the inclusion and exclusion criteria, a total of 50 morbid obese patients (BMI $>35 \mathrm{~kg} / \mathrm{m}^{2}$ ) aged $20-40$ years diagnosed with T2DM of less than 10-year duration were included in the study. Out of these, 19 were females and 31 were males (Table 1). Majority of study subjects $(n=37)$ were in the age range $30-40$ years whereas 19 subjects were in the age group 20-30 years (Table 2).

Statistical analysis showed that the data were normally distributed; therefore, a parametric test of significance (ANOVA) was applied.

There was a significant decrease in fasting plasma glucose levels at 9 weeks and 12 weeks follow-up after SG as compared to levels before surgery (Table 3). This improved glycemia was accompanied with an improvement in insulin sensitivity (as evident from a decrease in HOMA-IR values, which was statistically significant) (Table 3).

Table 1: Distribution of patients according to gender

\begin{tabular}{lll}
\hline Gender & Frequency $(n)$ & Percentage \\
\hline Male & 31 & 62 \\
Female & 19 & 38 \\
\hline
\end{tabular}

Table 2: Distribution of patients according to age

\begin{tabular}{lll}
\hline Age (years) & Frequency $(n)$ & Percentage \\
\hline $20-30$ & 13 & 26 \\
$31-40$ & 37 & 74 \\
\hline
\end{tabular}

\section{Discussion}

This study aimed at analyzing the effect of SG on fasting plasma glucose and insulin resistance in morbidly obese patients with T2DM. The results revealed a significant improvement in glycemia and insulin sensitivity as observed at 9-week and 12-week follow-up visits after SG (Table 3). These results are in agreement with the findings of previous studies conducted in bariatric surgery cases. ${ }^{2,13-20}$

One of the largest, longest-running prospective, nonrandomized, interventional trial, the Swedish Obesity Subjects Study (SOSS), ${ }_{1}^{17}$ examined the effects of bariatric surgery on the glycemic status of about 4,000 obese patients over a 2-year follow-up. They observed a $60 \%$ decrease in plasma insulin and a $20 \%$ decrease in plasma glucose in patients undergoing surgery as compared to the conventionally treated controls. Several other authors like Hady et al., ${ }^{20}$ Aminian et al., ${ }^{15}$ and Hutter et al. ${ }^{16}$ also reported similar decrease in the blood glucose levels in short- and medium-term follow-up after undergoing SG.

The literature regarding the effect of SG on insulin homeostasis is sparse and is limited to a follow-up duration up to 1 year post surgery. ${ }^{18,21-23}$ Lee et al. ${ }^{18}$ and Casella et al..$^{22}$ reported a decrease in insulin resistance rather than an increase in insulin secretion at 1 year after SG whereas Rizzello et al. ${ }^{23}$ demonstrated a significant decrease in insulin resistance at 1 month after laparoscopic sleeve gastrectomy (LSG), which was independent of body mass reduction. Our study reveals similar results, showing an improvement in insulin resistance at 9 weeks and 12 weeks follow-up (Table 3).

The question of whether diabetes remission after bariatric surgery is mainly due to weight loss or weight loss-independent mechanisms remains controversial. Several other authors have postulated two main hypotheses, namely weight loss-based and weight loss-independent hormonal mechanism. 9,21,24 If glycemic improvement is due to diet control leading to weight loss, mechanisms involving control of appetite and energy balance are of importance; but if it involves mainly weight loss-independent effects, mechanisms of insulin secretion and its action on various organs should attract greater attention. ${ }^{9}$

Number of studies focusing on long-term effects are ongoing, ${ }^{5,15,25,26}$ but very few data are available on the very early weight-independent effects of SG in patients with obesity and T2DM. In comparison to purely restrictive bariatric procedures such as gastric banding, glycemic control after SG often occurs before significant weight loss. ${ }^{21}$ This finding suggests that control of the glycemic status may be a direct effect of the surgery rather than a consequence of weight loss. ${ }^{21}$ Very low-calorie diets can significantly lower blood glucose and increase hepatic insulin sensitivity even before weight loss begins. ${ }^{9}$ As little as $5 \%$ weight loss over a period of 4 years was highly effective for diabetes remission as shown in the Diabetes Prevention Program. ${ }^{27}$

Although the study by Abu-Gazala et al. ${ }^{28}$ points to changes in hepatic insulin sensitivity, exact mechanisms of weight

Table 3: Comparison of glycemic indices and insulin resistance in type 2 diabetic morbidly obese patients: presurgery, at 9 weeks and 12 weeks follow-up after surgery

\begin{tabular}{lcccc}
\hline Parameter & Presurgery $($ mean $\pm S D)$ & 9 weeks follow-up (mean $\pm S D)$ & 12 weeks follow-up $($ mean \pm SD) & $p$ value \\
\hline FPG $(\mathrm{mg} / \mathrm{dL})$ & $208.23 \pm 57.15$ & $192.5 \pm 53.28$ & $157.64 \pm 42.77$ & $0.000^{*}$ \\
S. insulin $(\mu \mathrm{lU} / \mathrm{mL})$ & $32.86 \pm 8.72$ & $28.41 \pm 6.43$ & $20.45 \pm 5.89$ & $0.000^{*}$ \\
HOMA-IR & $16.89 \pm 5.59$ & $13.5 \pm 4.82$ & $7.95 \pm 3.94$ & $0.000^{*}$ \\
\hline
\end{tabular}

FPG, fasting plasma glucose; S. insulin, fasting serum insulin; HOMA-IR, insulin resistance; ${ }^{*} p<0.05$, statistically significant using ANOVA as the parametric test 
loss-independent improvements in glycemic control after bariatric surgeries are still unknown. Several authors have postulated involvement of gut hormones like ghrelin and GLP-1, bile signaling, and changes in the gut microbiome. ${ }^{9,21,29}$ These factors have been suggested to influence the metabolic response after bariatric surgery but very few of these have been directly tested and confirmed so far. Sista et al. ${ }^{21}$ observed a decrease in ghrelin concentration and increased GLP-1 levels 3 days post SG and suggested that these gut hormones might play a pivotal role as ghrelin is known to inhibit glucose-induced insulin release leading to insulin resistance. Thus, removal of the gastric fundus might contribute to improved insulin sensitivity. On the other hand, the rapid transit of undigested food to the hind gut, due to enhanced gastric emptying after SG, seems to upregulate the secretion of GLP-1. Glucagon-like peptide 1 maintains blood glucose levels by upregulating insulin synthesis and proinsulin gene expression, as well as improving peripheral insulin sensitivity. ${ }^{22,23}$ Our study did not focus on hormonal aspects, hence contribution of these parameters could neither be excluded nor proven.

Lee et al. ${ }^{18}$ while defining remission of type Il diabetes as fasting plasma glucose (FPG) $<126 \mathrm{mg} / \mathrm{dL}$ and $\mathrm{HbA} 1 \mathrm{c}<6.5 \%$ without any glycemic therapy noticed remission of up to $50 \%$ at 1 -year follow-up of poorly controlled nonmorbidly obese T2DM patients undergoing LSG. They attributed this effect to the decreasing insulin resistance due to calorie restriction and weight loss rather than to enhanced insulin secretion.

In their systematic review and meta-analysis of T2DM after bariatric surgery, Buchwald et al. ${ }^{30}$ demonstrated complete resolution in $62 \%$ of patients even 2 years after surgery. Similarly, Abbatini et al. ${ }^{29}$ documented complete resolution in $73 \%$ of patients at 3 years of follow-up and improved glycemia in the rest. Neither the weight loss nor the degree of decrease in waist circumference was associated with resolution, suggesting that an additional mechanism may be involved.

While significant improvement in FPG and insulin sensitivity was a definitive finding in our study (Table 3), a complete remission of diabetes was not observed till 12 weeks of follow-up. This may suggest the need for a longer duration of follow-up and pave the way for future research as the exact mechanisms involved are still unclear and continue to attract researchers in this field.

\section{Conclusion}

The present study conducted in morbid obese patients with T2DM highlights the fact that the SG procedure showed early beneficial effects on the glycemic status as well as an improvement in the insulin sensitivity rather than an increased insulin secretion. As an extension to the present study, further studies involving correlation with anthropometry are going on at our center in obese and morbid obese patients in an attempt to analyze further the weight lossdependent and weight loss-independent hormonal mechanisms contributing to remission of T2DM in obesity.

\section{Strengths and Limitations}

The rare selection of SG patients as the study group adds to the novelty of this study. Inclusion of studying the biochemical parameters at two consecutive follow-up visits and comparing them with the baseline presurgical values strengthens the reliability of the study results.

A possible limitation could be the relatively smaller sample size and a short follow-up period. The weight loss could not be statistically evaluated due to loss of follow-up data on anthropometry after surgery.

\section{Future Scope of the Study}

Further longitudinal studies in a larger sample size including estimation of postprandial glucose and $\mathrm{HbA1c}$ are desirable to confirm these results.

\section{Clinical Significance}

Interpretation of the improved glycemic status in this study along with an understanding of the exact mechanisms underlying this significant improvement could not only provide an insight into the pathophysiology of "diabesity" but also assist in the development of a better therapeutic approach for long-term remission of diabetes in obese patients.

\section{References}

1. Midha $T$, Nath B, Kumari R, et al. Prevalence and determinants of obesity in the adult population of Kanpur district -- a populationbased study. J Indian Med Assoc 2011;109(8):538-542.

2. Noria SF, Grantcharov T. Biological effects of bariatric surgery on obesity-related comorbidities. Can J Surg 2013;56(1):47-57. DOI: 10.1503/cjs.036111.

3. O'Brien PE. Bariatric surgery: mechanisms, indications, and outcomes. J Gastroenterol Hepatol 2010;25(8):1358-1365. DOI: 10.1111/j.14401746.2010.06391.x.

4. Franz MJ, Van Wormer JJ, Crain AL, et al. Weight-loss out comes: a systematic review and meta-analysis of weight-loss clinical trials with a minimum 1-year follow-up. J Am Diet Assoc 2007;107(10):1755-1767. DOI: 10.1016/j.jada.2007.07.017.

5. Schauer PR, Bhatt DL, Kirwan JP, et al. Bariatric surgery versus intensive medical therapy for diabetes - 5-year outcomes. N Engl J Med 2017;376(7):641-651. DOI: 10.1056/NEJMoa1600869.

6. Dadan J, Iwacewicz P, Hady HR. New approaches in bariatric surgery. Videosurgery Miniinv 2008;3:66-70.

7. Avenell A, Brown TJ, McGee MA, et al. What are the long-term benefits of weight reducing diets in adults? A systematic review of randomized controlled trials. J Hum Nutr Diet 2004;17(4):317-335. DOI: 10.1111/j.1365-277X.2004.00531.x.

8. Bose M, Oliván B, Teixeira J, et al. Do incretins play a role in their mission of type 2 diabetes after gastric bypass surgery: what are the evidence? Obes Surg 2009;19(2):217-229. DOI: 10.1007/s11695-0089696-3.

9. Kirwan JP, Münzberg H, Berthoud HR. Mechanisms responsible for metabolic improvements of bariatric surgeries. Diabetes 2018;67(6):1043-1044. DOI: 10.2337/dbi18-0011.

10. Valderas JP, Irribarra V, Rubio L, et al. Effects of sleeve gastrectomy and medical treatment for obesity on glucagon-like peptide 1 levels and glucose homeostasis in non-diabetic subjects. Obes Surg 2011;21(7):902-909. DOI: 10.1007/s11695-011-0375-4.

11. Langer FB, Hoda R, Bohdjalian A, et al. Sleeve gastrectomy and gastric banding: effects on plasma ghrelin levels. Obes Surg 2005;15(7): 1024-1029. DOI: 10.1381/0960892054621125.

12. Dixon JB, le Roux CW, Rubino F, et al. Bariatric surgery for type 2 diabetes. Lancet 2012;379(9833):2300-2311. DOI: 10.1016/S01406736(12)60401-2.

13. Azizi F. Bariatric surgery for obesity and diabetes. Arch Iran Med 2013;16(3):182-186. DOI: 013163/AIM.0012.

14. Schauer PR, Kashyap SR, Wolski K, et al. Bariatric surgery versus intensive medical therapy in obese patients with diabetes. $N$ Engl J Med 2012;366(17):1567-1576. DOI: 10.1056/NEJMoa1200225.

15. Aminian A, Brethauer SA, Andalib A, et al. Can sleeve gastrectomy "cure" diabetes? Long-term metabolic effects of sleeve gastrectomy in patients with type 2 diabetes. Ann Surg 2016;264(4):674-681. DOI: 10.1097/SLA.0000000000001857. 
16. Hutter MM, Schirmer BD, Jones DB, et al. First report from the American College of Surgeons Bariatric Surgery Center Network: laparoscopic sleeve gastrectomy has morbidity and effectiveness positioned between the band and the bypass. Ann Surg 2011;254(3):410-420, discussion 420-422 10.1097/SLA.0b013e31822c9dac.

17. Sjöström L. Review of the key results from the Swedish Obese Subjects (SOS) trial - a prospective controlled intervention study of bariatric surgery. J Intern Med 2013;273(3):219-234. DOI: 10.1111/joim.12012.

18. Lee WJ, Ser KH, Chong K, et al. Laparoscopic sleeve gastrectomy for diabetes treatment in nonmorbidly obese patients: efficacy and change of insulin secretion. Surgery 2010;147(5):664-669. DOI: 10.1016/j.surg.2009.10.059.

19. Buchwald H, Avidor $Y$, Braunwald $E$, et al. Bariatric surgery: a systematic review and meta-analysis. JAMA 2004;292(14):1724-1737. DOI: 10.1001/jama.292.14.1724.

20. Hady HR, Dadan J, Gołaszewski P, et al. Impact of laparoscopic sleeve gastrectomy on body mass index, ghrelin, insulin and lipid levels in 100 obese patients. Wideochir Inne Tech Maloinwazyjne 2012;7(4):251-259. DOI: 10.5114/wiitm.2011.28979.

21. Sista F, Abruzzese V, Clementi M, et al. Resolution of type 2 diabetes after sleeve gastrectomy: a 2-step hypothesis. Surg Obes Relat Dis 2018;14(3):284-290. DOI: 10.1016/j.soard.2017.12.009.

22. Casella G, Soricelli E, Castagneto-Gissey L, et al. Changes in insulin sensitivity and secretion after sleeve gastrectomy. Br J Surg 2016;103(3):242-248. DOI: 10.1002/bjs.10039.

23. Rizzello M, Abbatini F, Casella G, et al. Early post-operative insulin resistance changes after sleeve gastrectomy. Obes Surg 2015;20(1):50-55. DOI: 10.1007/s11695-009-0017-2.
24. Rubino F. Bariatric surgery: effects on glucose homeostasis. Curr Opin Clin Nutr Metab Care 2006;9(4):497-507. DOI: 10.1097/01. mco.0000232914.14978.c5.

25. Helmiö M, Victorzon M, Ovaska J, et al. SLEEVEPASS: a randomized prospective multicenter study comparing laparoscopic sleeve gastrectomy and gastric bypass in the treatment of morbid obesity: preliminary results. Surg Endosc 2012;26(9):2521-2526. DOI: 10.1007/ s00464-012-2225-4.

26. Peterli R, Borbély Y, Kern B, et al. Early results of the Swiss Multicentre Bypass or Sleeve Study (SM-BOSS): a prospective randomized trial comparing laparoscopic sleeve gastrectomy and Roux-en-Y gastric bypass. Ann Surg 2013;258(5):690-694. DOI: 10.1097/ SLA.0b013e3182a67426.

27. Knowler WC, Barrett-Connor E, Fowler SE, et al. Reduction in the incidence of type 2 diabetes with lifestyle intervention or metformin. N Engl J Med 2002;346(6):393-403. DOI: 10.1056/ NEJMoa012512.

28. Abu-Gazala S, Horwitz E, Schyr RB, et al. Sleeve gastrectomy improves glycemia independent of weight loss by restoring hepatic insulin sensitivity. Diabetes 2018;67(6):1079-1085. DOI: 10.2337/db171028.

29. Abbatini F, Rizzello M, Casella G, et al. Long-term effects of laparoscopic sleeve gastrectomy, gastric bypass, and adjustable gastric banding on type 2 diabetes. Surg Endosc 2010;24(5): 1005-1010. DOI: 10.1007/s00464-009-0715-9.

30. Buchwald $\mathrm{H}$, Estok R, Fahrbach $\mathrm{K}$, et al. Weight and type 2 diabetes after bariatric surgery: a systematic review and meta-analysis. Am J Med 2009;122(3):248-256. DOI: 10.1016/j.amjmed.2008.09.041. 